\title{
Impact of intravenous alteplase on sub-angiographic emboli in high-resolution diffusion-weighted imaging following successful thrombectomy
}

\author{
Gabriel Broocks ${ }^{1}$ (D) Lukas Meyer $^{1} \cdot$ Reza Kabiri $^{1} \cdot$ Helge C. Kniep ${ }^{1} \cdot$ Rosalie McDonough ${ }^{1} \cdot$ Matthias Bechstein $^{1}$. \\ Noel van Horn ${ }^{1} \cdot$ Thomas Lindner $^{1}$ • Jan Sedlacik ${ }^{2}$ - Bastian Cheng ${ }^{3} \cdot$ Götz Thomalla $^{3}$ - Gerhard Schön ${ }^{4}$. Jens Fiehler ${ }^{1}$. \\ Uta Hanning ${ }^{1}$ • Michael H. Schönfeld ${ }^{1,5}$
}

Received: 8 January 2021 / Revised: 21 March 2021 / Accepted: 1 April 2021 / Published online: 8 May 2021

(C) The Author(s) 2021

\begin{abstract}
Objective Thrombus microfragmentation causing peripheral emboli (PE) during mechanical thrombectomy (MT) may modulate treatment effects, even in cases with successful reperfusion. This study aims to investigate whether intravenous alteplase is of potential benefit in reducing PE after successful MT.

Methods Patients from a prospective study treated at a tertiary care stroke center between 08/2017 and 12/2019 were analyzed. The main inclusion criterion was successful reperfusion after MT (defined as expanded thrombolysis in cerebral infarction (eTICI) scale $\geq$ 2b50) of large vessel occlusion anterior circulation stroke. All patients received a high-resolution diffusion-weighted imaging (DWI) follow-up $24 \mathrm{~h}$ after MT for PE detection. Patients were grouped as "direct MT" (no alteplase) or as MT plus additional intravenous alteplase. The number and volume of ischemic core lesions and PE were then quantified and analyzed.

Results Fifty-six patients were prospectively enrolled. Additional intravenous alteplase was administered in 46.3\% (26/56). There were no statistically significant differences of PE compared by groups of direct MT and additional intravenous alteplase administration regarding mean numbers $(12.1,95 \%$ CI $8.6-15.5$ vs. $11.1,95 \%$ CI $7.0-15.1 ; p=0.701)$, and median volume (0.70 mL, IQR 0.21-1.55 vs. $0.39 \mathrm{~mL}$, IQR 0.10-1.62; $p=0.554)$. In uni- and multivariable linear regression analysis, higher eTICI scores were significantly associated with reduced PE, while the administration of alteplase was neither associated with numbers nor volume of peripheral emboli. Additional alteplase did not alter reperfusion success.

Conclusions Intravenous alteplase neither affects the number nor volume of sub-angiographic DWI-PE after successful endovascular reperfusion. In the light of currently running randomized trials, further studies are warranted to validate these findings.

Key Points

- Thrombus microfragmentation during endovascular stroke treatment may cause peripheral emboli that are only detectable on diffusion-weighted imaging and may directly compromise treatment effects.

- In this prospective study, the application of intravenous alteplase did not influence the occurrence of peripheral emboli detected on high-resolution diffusion-weighted imaging.

- A higher degree of recanalization was associated with a reduced number and volume of peripheral emboli and better functional outcome, while contrariwise, peripheral emboli did not modify the effect of recanalization on modified Rankin Scale scores at day 90 .
\end{abstract}

Gabriel Broocks and Lukas Meyer contributed equally to this work.

Gabriel Broocks

g.broocks@uke.de

1 Department of Diagnostic and Interventional Neuroradiology, University Medical Center Hamburg-Eppendorf, Martinistrasse 52, 20246 Hamburg, Germany

2 Centre for the Developing Brain \& Biomedical Engineering Department, School of Biomedical Engineering \& Imaging Sciences, King's College London, London, UK
3 Department of Neurology, University Medical Center Hamburg-Eppendorf, Hamburg, Germany

4 Institute of Epidemiology and Medical Biometry, University Medical Center Hamburg-Eppendorf, Hamburg, Germany

5 Department of Neuroradiology, University Köln, Köln, Germany 
Keywords Stroke $\cdot$ Thrombectomy $\cdot$ Magnetic resonance imaging $\cdot$ Ischemia $\cdot$ Infarction

$\begin{array}{ll}\text { Abbreviations } \\ \text { ASPECTS } & \text { Alberta Stroke Program Early CT Score } \\ \text { CI } & \text { Confidence interval } \\ \text { DSA } & \text { Digital subtraction angiography } \\ \text { DWI } & \text { Diffusion-weighted imaging } \\ \text { eTICI } & \text { Expanded thrombolysis in cerebral infarction } \\ \text { IQR } & \text { Interquartile range } \\ \text { LVO } & \text { Large vessel occlusion } \\ \text { MRI } & \text { Magnetic resonance imaging } \\ \text { mRS } & \text { Modified Rankin scale } \\ \text { MT } & \text { Mechanical thrombectomy } \\ \text { NIHSS } & \text { National Institute of Health Stroke Scale } \\ \text { PE } & \text { Peripheral emboli }\end{array}$

\section{Introduction}

Mechanical thrombectomy (MT) is of benefit to patients with acute ischemic stroke and large vessel occlusion (LVO) in the anterior circulation [1]. Although intravenous (IV) thrombolysis with alteplase, a recombinant tissue plasminogen activator, is the standard medical treatment for acute ischemic stroke patients, it is still ambiguous whether its application prior MT improves functional outcome in patients with LVO compared to direct MT [2]. It is well established that the key for improving functional outcome is successful revascularization, as demonstrated in multiple trials [3, 4]. IV lysis alone, however, rarely leads to sufficient recanalization in LVO stroke and might increase the risk of secondary hemorrhage $[5,6]$. In the light of the recently published randomized controlled trial "DIRECT MT" [7] that observed non-inferiority of direct MT compared to MT with bridging IV lysis, it becomes even more relevant to understand the effect of alteplase on ischemic lesion pathophysiology $[7,8]$.

Potential advantages of IV lysis may include improved microvascular reperfusion and lysis of periperhal emboli caused by thrombus (micro)fragmentation during MT [9]. Recently, it has been reported that the so-called subangiograpic peripheral emboli (PE) can be detected on highresolution diffusion-weighted imaging (DWI), including cases in which complete angiographic reperfusion was achieved [10]. Accordingly, bridging IV alteplase prior to MT might mediate the occurrence of PE; however, this hypothesis has yet to be investigated. This prospective cohort study analyzes the impact of IV alteplase on PE after successful MT. We hypothesized that the administration of bridging alteplase prior to MT is associated with smaller numbers and/or volumes of postinterventional PE detectable on high-resolution DWI compared to direct MT.

\section{Material and methods}

\section{Study population}

Patient data was collected in a prospective non-randomized cohort study treated at a tertiary care stroke center between 08/2017 and $12 / 2019$. Inclusion criteria were defined as (1) CTangiography confirmed proximal LVO anterior circulation stroke without signs of dissection, (2) treated with MT, leading to (3) successful reperfusion defined as an expanded Thrombolysis in Cerebral Infarction (eTICI) score $\geq 2 b 50$ [11]. The study was approved by the local ethics committee (Ethics Committee of the Physician Board Hamburg; approval number WF 019/19) and all patients gave written informed consent (or by authorized family members/ legal guardians) according to the guidelines of the local ethics committee. This study was conducted in accordance with the Declaration of Helsinki and the STROBE guidelines. The standard imaging modality at baseline was computed tomography, with the intention not to delay endovascular treatment, in line with current guideline recommendations [12].

\section{Procedure}

If eligible, all patients admitted up to $4.5 \mathrm{~h}$ after symptom onset received bridging IV alteplase $(0.9 \mathrm{mg}$ per $\mathrm{kg}$ body weight) prior to MT according to German guidelines [13]. Procedures were performed under general anesthesia or conscious sedation. Choice of access material and thrombectomy devices was at the discretion of the acting neurointerventionalist in charge.

\section{Clinical data}

Baseline demographic data, National Institutes of Health Stroke Scale (NIHSS) score on admission and discharge, and modified Rankin scale (mRS) scores at 90-days followup were documented and analyzed.

\section{Follow-up imaging and analysis}

Postinterventional digital subtraction angiography (DSA) imaging was evaluated using the eTICI score, as proposed by Liebeskind et al [14]. Follow-up MRI was performed on a 1.5T MRI scanner (Siemens Magnetom Avanto) within $24 \mathrm{~h}$ following intervention and included axial whole-brain high-resolution DWI using single-shot, multi-slice, spin-echo, echoplanar imaging sequences with diffusion gradients in three orthogonal directions and the following parameters: TR/TE $13,000 / 90 \mathrm{~ms}, b$ values of 0,500 , and $1000 \mathrm{~s} / \mathrm{mm}^{2}$, matrix $192 \times 192$, no gap, field of view 240 , slice thickness $2 \mathrm{~mm}$, 


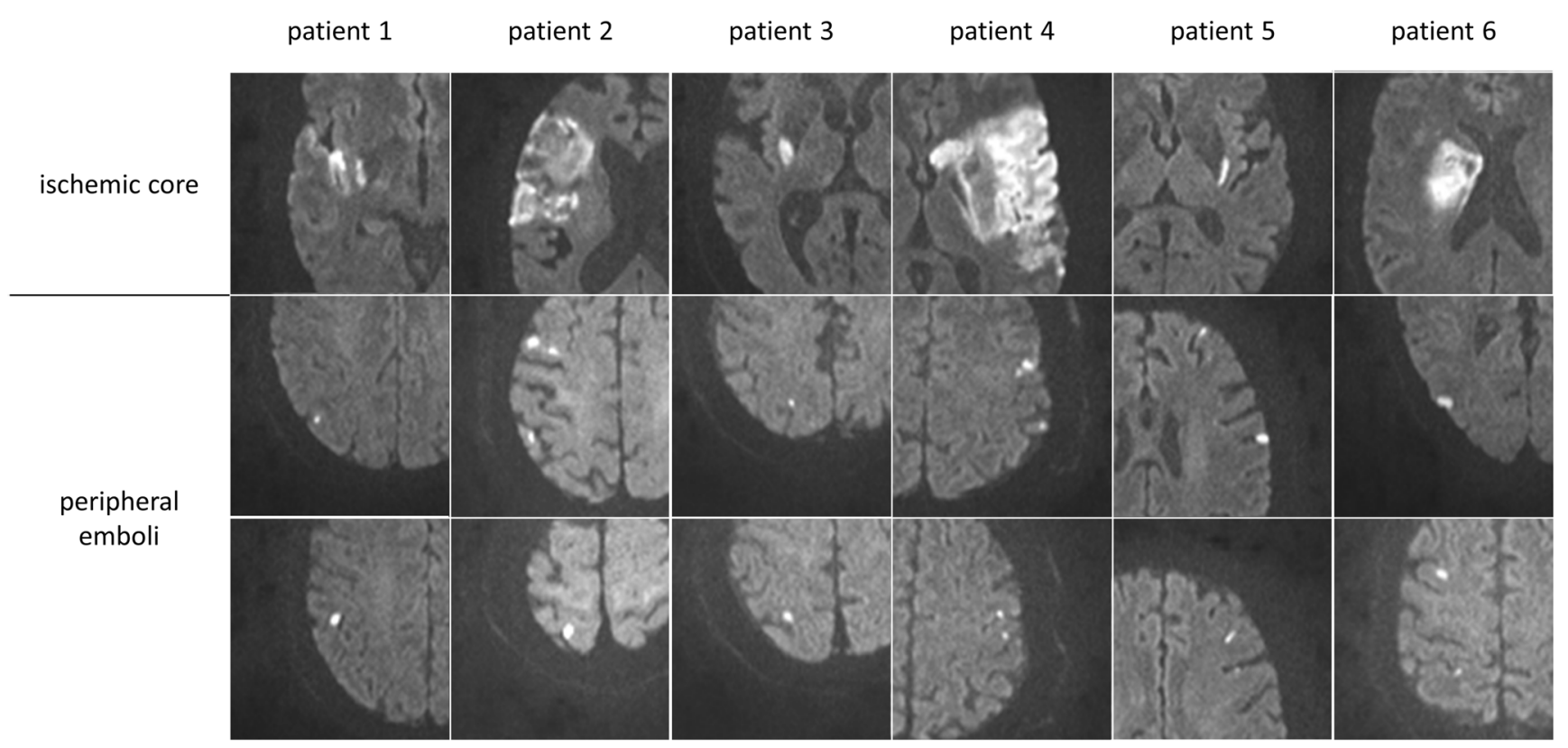

Fig. 1 Example of six patients with ischemic cores (upper row) and peripheral emboli (lower rows) on the trace images of the high-resolution DWI

acquisition time of $457 \mathrm{~s}$. The spatial resolution in the $3 \mathrm{D}$ volume was increased, in addition to increasing both the TR and TE and the number of excitations substantially to improve the signal-to-noise ratio and to reduce the occurrence of sequence-related artifacts (see Supplemental Figure III). PE were detected as visually evident focal diffusion restrictions with a high signal on the trace images with a $b$ value of 1000 and a reduced ADC-value on the ADC maps distal to a continuous core high-resolution DWI lesion (Fig. 1). DWI lesions were segmented semi-automatically with the Analyze 11.0 software package (AnalyzeDirect, Inc.) using seed-growing algorithms. Subsequently, the total PE volume in milliliters was derived automatically in Analyze using the sampling option. DSA and MRI analyses were performed independently by two readers who did not participate in the endovascular therapy. The imaging analysis was performed blinded to any clinical data including treatment. Discrepancies between readers were resolved by consensus in a joint side-by-side comparison of segmented lesion maps and joint selection and manual selection of the appropriate lesion maps.

\section{Statistical analysis}

Standard descriptive statistics were applied for all data. Patients who received IV lysis were compared to patients without IV lysis (Table 1). Wilcoxon signed rank test was performed to compare continuous variables, as appropriate. The chi-square test and Fisher's exact test were used to compare categorial variables. A subgroup analysis for patients with a time from symptom onset to reperfusion $\leq 6 \mathrm{~h}$ was performed. Furthermore, the association of IV alteplase and number/volume of peripheral emboli was analyzed using
Table 1 Overview of patients' baseline characteristics, and comparison grouped by IV alteplase administration

\begin{tabular}{lllll}
\hline Baseline characteristics & $\begin{array}{l}\text { All patients } \\
(n=56)\end{array}$ & $\begin{array}{l}\text { IV alteplase } \\
(n=26)\end{array}$ & $\begin{array}{l}\text { No IV alteplase } \\
(n=30)\end{array}$ & $\begin{array}{l}p \\
\text { value }\end{array}$ \\
\hline Age in years, median (IQR) & $71.5(63-81)$ & $71.5(66.75-81)$ & $71(62-82.25)$ & 0.837 \\
Female sex, $n(\%)$ & $26(46.4)$ & $10(38.5)$ & $16(53.3)$ & 0.266 \\
Atrial fibrillation, $n(\%)$ & $24(42.9)$ & $12(46.2)$ & $20(66.7)$ & 0.122 \\
Admission NIHSS, median (IQR) & $13.5(10-16.75)$ & $12.5(10-17)$ & $14(9.75-16.25)$ & 0.921 \\
Occlusion site, $n(\%)$ & & & & \\
Tandem occlusion & $9(16.1)$ & $3(5.4)$ & $6(10.7)$ & 0.481 \\
Terminal ICA & $12(21.4)$ & $6(10.7)$ & $6(10.7)$ & 0.521 \\
MCA & $43(76.8)$ & $19(33.9)$ & $24(42.9)$ & \\
ACA & $1(1.8)$ & $1(1.8)$ & 0 & $8(6-9)$ \\
ASPECTS, median (IQR) & $8(6-9)$ & $9(6-10)$ & & 0.073 \\
\hline
\end{tabular}


univariable and multivariable linear regression analysis with eTICI, ASPECTS, time from initiation of IV alteplase to reperfusion, and age as independent parameter using backwards selection.

The two-way analysis of variance (ANOVA) was performed to investigate the effect of alteplase on peripheral emboli according to the reperfusion grade (dependent variable: number of $\mathrm{PE}$ and volume of PE; with reperfusion grade according to eTICI, and application of alteplase as independent variables; see supplemental content).

Equivalence tests were performed to further investigate whether there is a significant difference comparing the number and volume of peripheral emboli in patients with versus without IV alteplase (see supplemental content).

Finally, the impact of intravenous alteplase on functional outcome, as well as the association of PE and functional outcome, was investigated using univariable and multivariable ordinal regression analysis.

Statistics were calculated using SPSS 24.0 (IBM SPSS Statistics for Windows: IBM Corp.) and StataSE (StataCorp). $p$ values $<0.05$ were considered statistically significant.

\section{Results}

\section{Patient population}

Fifty-six patients met the inclusion criteria and were prospectively enrolled and analyzed. The median age was 71.5 years (IQR 63-81) and 46.4\% (26/56) were female. Median ASPECTS was 8 (6-9) and median NIHSS was 13.5 (10$16.75)$ on admission. A total of $46.4 \%$ (26/56) of all patients received IV alteplase prior to MT. The mean time from symptom onset to imaging was $2.8 \mathrm{~h}$ (SD: 1.0) in patients with bridging IV alteplase, which was shorter compared to patients with direct MT (mean time $5.4 \mathrm{~h}, \mathrm{SD}: 5.0$ ). The mean time from symptom onset to reperfusion was $6.5 \mathrm{~h}$ (SD: $3.0 \mathrm{~h}$ ). Table 1 provides an overview of patients' baseline characteristics, and comparison grouped by IV alteplase administration. Time from symptom onset to reperfusion was significantly longer ( $p=0.002)$ in the group of direct MT (370 min, IQR $249-574.5$ vs. $255 \mathrm{~min}$, IQR 201-338.5). There were no significant differences in the distribution of reperfusion grades (7/56 eTICI 2b50; 17/56 eTICI 2b67; 12/56 eTICI 2c; 20/56 eTICI 3 ) between the two groups ( $p=0.740)$.

Three out of 56 patients $(5.3 \%)$ showed secondary intracranial hemorrhage on follow-up imaging (as stated in the MRI report, which is compiled by an experienced boardcertified neuroradiologist and verified by a further experienced attending neuroradiologist). None of these patients fulfilled criteria of a symptomatic hemorrhage (defined as NIHSS increase $>4$, NIHSS increase $>2$ points in one category, clinical deterioration leading to intubation, hemicraniectomy, or other major medical/surgical intervention).

Impact of intravenous alteplase on peripheral emboli

The mean number of PE was 11.6 (95\% CI 9.1-14.2) and did not differ significantly between those with direct MT and those with additional IV alteplase numbers $(12.1,95 \% \mathrm{CI}$ 8.6-15.5 vs. $11.195 \%$ CI $7.0-15.1 ; p=0.701$, respectively). In all patients, the median volume of the core infarct was $19.33 \mathrm{~mL}$ (IQR 6.08-54.51) and the median volume of all PE was $0.62 \mathrm{~mL}$ (IQR 0.12-1.57). A volumetric-based comparison of the study groups showed no significant differences in all patients or the subgroup of patients with a time from symptom onset to reperfusion $\leq 6 \mathrm{~h}$ (Fig. 2). In the cohort of patients receiving additional alteplase, no significant relationship between the time from initiation of IV alteplase to reperfusion and number $(p=0.833)$ or volume of $\operatorname{PE~}(p=0.871)$ was observed.

In univariable linear regression analysis, IV alteplase was not significantly associated number of $\mathrm{PE}(\beta:-0.99,95 \% \mathrm{CI}$ : -6.1 to $4.2 ; p=0.7)$ or volume of $\mathrm{PE}(\beta:-130.9,95 \% \mathrm{CI}:-$ 957.3 to $695.5 ; p=0.8)$. In multivariable linear regression analysis, IV alteplase was neither associated with number $(\beta$ : -1.6 , SD: $2.1 ; p=0.45)$ nor volume of PE $(\beta:-133.5$, SD: $422.7 ; p=0.75)$. The degree of reperfusion indicated by eTICI was the only remaining variable to be significantly and independently associated with the number of $\operatorname{PE~}(\beta:-5.6, \mathrm{SD}: 1.0$; $p<0.0001)$, and volume of $\mathrm{PE}(\beta:-371.8$, SD: 187.5 ; $p=0.04)$, adjusted for ASPECTS, age, and application of IV alteplase. Table 2 provides an overview and comparison of procedural and functional outcome together with the results of the imaging analysis according to IV alteplase administration.

Two ANOVAs investigating the relationship of reperfusion and IV alteplase on number and volume of PE can be found in the supplemental material. We observed a significantly decreasing number of $\mathrm{PE}$ and by trend reduced volume of PE with higher reperfusion grades, while IV alteplase was neither associated with different number nor volume of $\mathrm{PE}$ for every reperfusion grade with significantly overlapping confidence intervals ( $p=0.24 ; p=0.65)$. (supplemental material).

Equivalence tests demonstrated that there is no association between the application of IV alteplase and the number or volume of PE. Based on a tolerated range of $\pm 1 \mathrm{PE}$ number or $\pm 0.13 \mathrm{~mL} \mathrm{PE}$ volume, respectively, equivalence may be assumed.

In univariable und multivariable ordinal regression analysis, there was no association between the application of alteplase and functional outcome $(p>0.1)$. Significant predictors of outcome were age (odds ratio for mRS shift: 1.06, $p<0.02$ ) and ASPECTS (OR: 0.55, $p=0.001$ ). Second, we 
Fig. 2 Box plots and means (red crosses) with $95 \%$ confidence interval (red whiskers) of numbers (light blue) and volume (dark blue) of peripheral emboli compared by patients treated with $(n=21)$ and without $(n=14)$ additional intravenous alteplase in patients with a time from symptom onset to reperfusion $\leq 6 \mathrm{~h}$

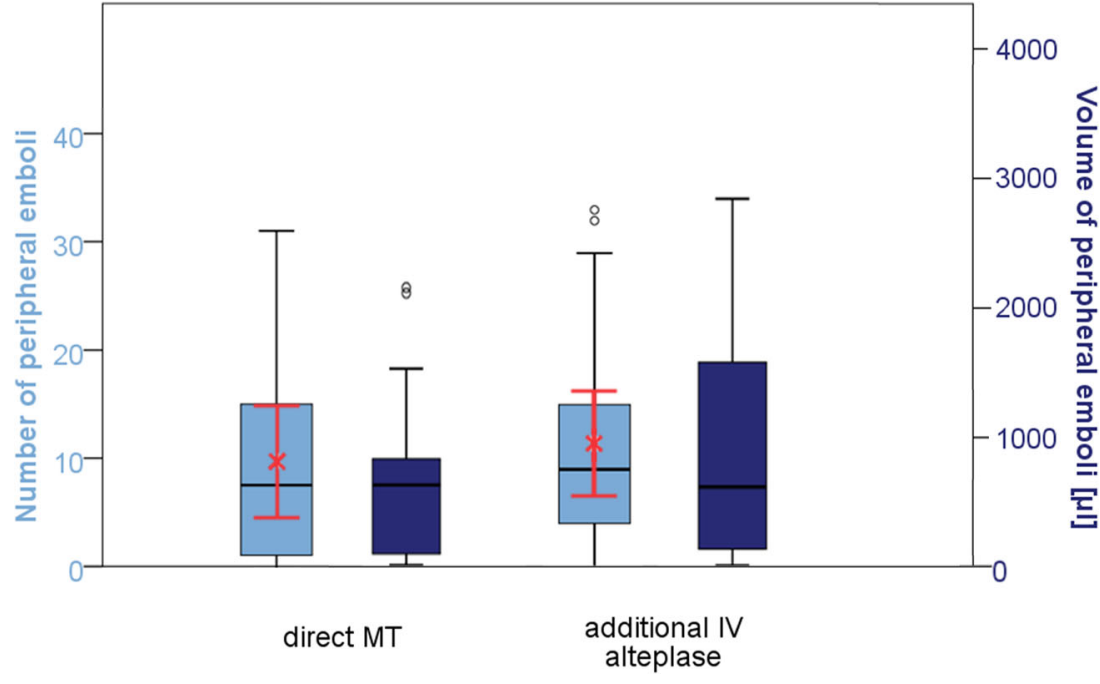

investigated whether the volume or number of PE is associated with functional outcome. Neither number nor volume of peripheral emboli was significantly associated with the mRS at day 90 in this patient cohort $(p=0.95, p=0.78)$. The volume of the DWI core lesion was significantly associated with outcome (odds ratio for mRS shift per $\mathrm{mL}: 1.02,95 \% \mathrm{CI}$ : 1.01-1.04, $p<0.001)$.

Finally, we analyzed the effect of intravenous alteplase on the functional outcome according to the baseline core lesion volume and investigated whether there is an interaction between intravenous lysis and core lesion volume. While core lesion volume was significantly associated with functional outcome (OR: 0.97, 95\% CI: 0.95-0.99, $p=0.008$; Supplemental Figure IV), there was no effect of intravenous lysis $(p>0.6)$, and the interaction term was not significant $(p=0.82)$.

\section{Discussion}

Several randomized controlled trials are currently investigating the potential clinical benefit or harm of additional IV alteplase administration prior to thrombectomy (MR CLEAN- NO IV[15], SWIFT DIRECT, clinical trial ID: NCT03192332). Our data suggest that additional IV alteplase has no relevant impact on sub-angiographic peripheral DWI emboli in cases of successful reperfusion (eTICI $\geq 2 \mathrm{~b} 50$ ).

Iatrogenic embolization attributed to MT represents a feared complication, potentially irreversibly compromising

Table 2 Comparison of procedural and functional outcome, and imaging analysis between patients treated with and without intravenous alteplase

\begin{tabular}{|c|c|c|c|c|}
\hline Procedural and functional outcome & All patients $(n=56)$ & IV alteplase $(n=26)$ & No IV alteplase $(n=30)$ & $p$ value \\
\hline eTICI (2b50/2b67/2c/3) & $7 / 17 / 12 / 20$ & $4 / 8 / 4 / 10$ & $3 / 9 / 8 / 10$ & 0.740 \\
\hline $\begin{array}{l}\text { Time symptom onset to reperfusion in min, } \\
\text { median (IQR) }[n=54]\end{array}$ & $281.5(230-465.5)$ & $255(201-338.5)$ & $370(249-574.5)$ & $0.022 *$ \\
\hline $\begin{array}{l}\text { Time IV alteplase administration to } \\
\text { reperfusion in min, median (IQR) }\end{array}$ & - & $148(104.75-205.25)$ & - & - \\
\hline mRS 90 days, median (IQR) & $2(0-3)[n=53]$ & $1.5(0-3)[n=26]$ & $2(1-4)[n=27]$ & 0.152 \\
\hline \multicolumn{5}{|l|}{ Imaging analysis } \\
\hline $\begin{array}{l}\text { Number of peripheral emboli, median } \\
\quad(\text { mean; } 95 \% \mathrm{CI})\end{array}$ & $11.6(9.1-14.2)$ & $11.1(7.0-15.1)$ & $12.1(8.6-15.5)$ & 0.701 \\
\hline Time from symptom onset to reperfusion $\leq 6 \mathrm{~h}$ & $10.6(7.2-14.0)[n=35]$ & $11.3(7.0-15.6)[n=21]$ & $9.6(4.4-14.9)[n=14]$ & 0.413 \\
\hline Volume core infarct in mL, median (IQR) & $19.33(6.08-54.51)$ & $15.68(5.69-51.48)$ & $25.15(6.04-56.55)$ & 0.501 \\
\hline Time from symptom onset to reperfusion $\leq 6 \mathrm{~h}$ & $17.26(3.48-48.71)[n=35]$ & $18.05(5.30-74.16)[n=21]$ & $5.97(2.03-33.27)[n=14]$ & 0.281 \\
\hline Volume of peripheral infarcts in $\mathrm{mL}$, median (IQR) & $0.62(0.12-1.57)$ & $0.39(0.10-1.62)$ & $0.70(0.21-1.55)$ & 0.554 \\
\hline Time from symptom onset to reperfusion $\leq 6 \mathrm{~h}$ & $0.62(0.10-1.53)[n=35]$ & $0.62(0.10-1.65)[n=21]$ & $0.63(0.08-1.01)[n=14]$ & 0.590 \\
\hline
\end{tabular}

*significant $(p<0.05)$ 
reperfusion success and patient outcome [16]. In a recent study, it has been observed that some of these emboli are not detectable on DSA but can be demarcated on follow-up MRI with high-resolution DWI imaging [10]. Depending on the volume, and perhaps more importantly the topography, these emboli could have a high impact on post-stroke outcome and cognitive recovery or decline $[17,18]$. In the current debate concerning whether or not IV alteplase should be administered prior to MT, one of the main arguments for the additional pharmacological treatment is the possible improvement of microvascular reperfusion as well as lysis of distal emboli, generally seen as a potential complication following endovascular reperfusion [19]. Recently, the first randomized controlled trials on direct MT versus MT plus additional IV alteplase have been published showing that direct MT was noninferior with regard to functional outcome [7]. In this context, the present study further corroborates these findings using a different metric. Investigating the influence of IV alteplase on sub-angiographic emboli, we neither observed differences in the prevalence of PE $24 \mathrm{~h}$ after endovascular treatment comparing patients with direct MT and those with additional IV alteplase, nor did we see a time-dependent effect in patients that received IV alteplase. The relevance of these findings is independent from the missing pre-procedural MRI studies, required to confirm the periprocedural nature of the PE. Although this missing data represents a limitation of this study, a positive effect of IV alteplase would be expected regardless if the origin of microemboli was pre-procedural or periprocedural [18]. Furthermore, the volume of PE in both groups with and without IV alteplase did not differ significantly. Consequently, additional IV alteplase neither led to reduced numbers nor did it reduce the size of the peripheral embolic lesions. Because our analysis only included cases with successful reperfusion (TICI $\geq 2 \mathrm{~b} 50$ ), this finding suggests that additional IV alteplase has no strong effect on the smaller peripheral ischemic lesions following endovascular reperfusion. Likewise, the volume of core lesions did not differ significantly, confirming prior study results that the cumulative effect of IV lysis is likely marginal in cases with proximal LVO, thereby requiring a higher number needed to treat $[19,20]$. In corroboration results of previous studies, there were no differences observed in the degree of successful reperfusion and thus, direct MT seems to be as effective as MT plus additional IV lysis [19]. The probability of a type II error, i.e., falsely missing a strong effect of IV alteplase on the incidence of peripheral emboli, was very low in our study. Nevertheless, we cannot conclude that IV alteplase is per se ineffective for LVO stroke since bridging therapy can in some cases lead to successful reperfusion before the patient even reaches an endovascular stroke center. Furthermore, a potential treatment effect of IV alteplase might lie beyond the observable volume of infarcted tissue [20].
In our cohort, the functional outcome at 90 days was comparably good between both groups. Accordingly, the clinical importance of microembolic ischemic lesions remains unclear. Their impact most likely lies outside the scope of the most commonly used global functional outcome measures like the mRS and could involve rather subtle differences in neurological dysfunction [21]. Further research could investigate whether PE have an effect on NIHSS scores stratified by symptoms, or with further neurological assessment scores that represent more subtle cognitive changes. Accordingly, Kaesmacher et al showed recently that infarcts larger than $2 \mathrm{~cm}$ distal to the occlusion in initially not the hypoperfused territory and new territories are associated with poor outcome [22]. Further studies are warranted to investigate the impact of interventional techniques and devices with regard to thrombus characteristics on the occurrence of PE after MT and their influence on outcome [16, 23]. Moreover, further studies might investigate the impact of intravenous alteplase on PE in patients without or failed endovascular recanalization, considering that the present study only analyzed patients with successful endovascular recanalization.

\section{Limitations}

This prospective observational study is subject to all limitations associated with a non-randomized study design. The number of patients was relatively small due to strict inclusion criteria intending to obtain a homogenous patient cohort. Patients were required to undergo MRI examination without significant delay of diagnosis, or significant logistic efforts. Hence, patients who have showed significant clinical complications such as severe and/or sudden neurological deterioration leading to ineligibility of MR imaging, or requiring rapid imaging to exclude severe secondary parenchymal hemorrhage, could not be included in this study, which potentially affects patients who suffered from symptomatic intracranial hemorrhage. Furthermore, since MRI imaging was not a part of the initial stroke imaging protocol for suspected LVO stroke, evidence is missing to confirm the periprocedural nature of all $\mathrm{PE}$. Hence, patients receiving both IV lysis plus MT could have presented with an already higher number/volume of PE at baseline, which affects the evaluation of a protective effect of IV lysis in the follow-up imaging. However, it is important to point out that the patient groups did not show any significant differences in baseline and imaging parameters including atrial fibrillation. Second, a sensitive detection of PE in baseline imaging is not feasible, due to the long acquisition time of high-quality/high-resolution DWI (7.6 min in the present study). Although shorter high-resolution DWI sequences have been tested, these may not have sufficient resolution to detect and quantify small PE. In this cohort, 53\% of PE apparent on high-resolution DWI were missed in conventional DWI. While the utilization of MRI for acute stroke triage may have significant advantages (e.g., unknown onset stroke) and is used as a primary modality in several stroke centers [24, 25], CT 
is still the most widely performed initial imaging modality to detect LVO stroke, due to its applicability and availability [3, 8]. Although MRI was reported to be beneficial to guide IV lysis in WAKE-UP stroke patients, the positive effect of IV lysis on functional outcome in patients included in the WAKE-UP trial with an initial NIHSS $>10$, as observed in the majority of proximal LVO stroke, was unclear [26, 27]. Representing a realworld LVO patient cohort, the median NIHSS in this study was 14 (IQR: 10-17), and thus, the general use of MRI would have had highly affected general and personal clinical equipoise to include patients in this study [28].

\section{Conclusion}

Our study does not confirm the hypothesis that the additional administration of IV alteplase prior to mechanical thrombectomy leads to a significant post-procedural reduction in the number or volume of peripheral microembolic lesions visible on high-resolution DWI. Further studies are needed to investigate potential influencing factors of microemboli after thrombectomy, as well as their clinical impact.

Supplementary Information The online version contains supplementary material available at https://doi.org/10.1007/s00330-021-07980-0.

Acknowledgements Open Access funding enabled and organized by Projekt DEAL

Funding The authors state that this work has not received any funding.

\section{Declarations}

Guarantor The scientific guarantor of this publication is Gabriel Broocks.

Conflict of interest The authors of this manuscript declare relationships with the following companies:

Jens Fiehler: Consultant for Acandis, Boehringer Ingelheim, Codman, Microvention, Sequent, Stryker.

Speaker for Bayer Healthcare, Bracco, Covidien/ev3, Penumbra, Philips, Siemens. Grants from Bundesministeriums für Wirtschaft und Energie (BMWi), Bundesministerium für Bildung und Forschung, Deutsche Forschungsgemeinschaft (DFG), European Union, Covidien, Stryker (THRILL study), Microvention (ERASER study), Philips.

Götz Thomalla: Personal fees as consultant/lecturer 288 from Acandis, Bayer, Boehringer Ingelheim,Bristol-Myers Squibb/Pfizer, Daichi Sankyo, Stryker, and research grants from Bayer, BMWi, Corona-Foundation, DFG, Else Kröner-Fresenius Foundation, European Union (Horizon 2020), German Innovation Fund.

All other authors have no conflict of interest.

Statistics and biometry Gerhard Schön kindly provided statistical advice for this manuscript.

Informed consent Written informed consent was waived by the Institutional Review Board.

Ethical approval Institutional Review Board approval was obtained.

\section{Methodology}

- prospective

- observational

- performed at one institution

Open Access This article is licensed under a Creative Commons Attribution 4.0 International License, which permits use, sharing, adaptation, distribution and reproduction in any medium or format, as long as you give appropriate credit to the original author(s) and the source, provide a link to the Creative Commons licence, and indicate if changes were made. The images or other third party material in this article are included in the article's Creative Commons licence, unless indicated otherwise in a credit line to the material. If material is not included in the article's Creative Commons licence and your intended use is not permitted by statutory regulation or exceeds the permitted use, you will need to obtain permission directly from the copyright holder. To view a copy of this licence, visit http://creativecommons.org/licenses/by/4.0/.

\section{References}

1. Roman LS, Menon BK, Blasco J et al (2018) Imaging features and safety and efficacy of endovascular stroke treatment: a metaanalysis of individual patient-level data. Lancet Neurol 17:895-904

2. Turc G, Bhogal P, Fischer U et al (2019) European Stroke Organisation (ESO) - European Society for Minimally Invasive Neurological Therapy (ESMINT) Guidelines on Mechanical Thrombectomy in Acute Ischemic Stroke. J Neurointerv Surg. https://doi.org/10.1136/neurintsurg-2018-014569:neurintsurg2018-014569

3. Goyal M, Menon BK, van Zwam WH et al (2016) Endovascular thrombectomy after large-vessel ischaemic stroke: a meta-analysis of individual patient data from five randomised trials. Lancet 387: 1723-1731

4. Broocks G, Hanning U, Flottmann F et al (2019) Clinical benefit of thrombectomy in stroke patients with low ASPECTS is mediated by oedema reduction. Brain 142:1399-1407

5. Whiteley WN, Emberson J, Lees KR et al (2016) Risk of intracerebral haemorrhage with alteplase after acute ischaemic stroke: a secondary analysis of an individual patient data meta-analysis. Lancet Neurol 15:925-933

6. Bhatia R, Hill MD, Shobha N et al (2010) Low rates of acute recanalization with intravenous recombinant tissue plasminogen activator in ischemic stroke: real-world experience and a call for action. Stroke 41:2254-2258

7. Yang P, Zhang Y, Zhang L et al (2020) Endovascular Thrombectomy with or without intravenous alteplase in acute stroke. N Engl J Med 382:1981-1993

8. Broocks G, Leischner H, Hanning U et al (2020) Lesion age imaging in acute stroke: water uptake in CT versus dwi-FLAIR mismatch. Ann Neurol 88:1144-1152. https://doi.org/10.1002/ana. 25903

9. Kaesmacher J, Mordasini P, Arnold M et al (2019) Direct mechanical thrombectomy in tPA-ineligible and -eligible patients versus the bridging approach: a meta-analysis. J Neurointerv Surg 11: 20-27

10. Schonfeld MH, Kabiri R, Kniep HC et al (2020) Sub-angiographic peripheral emboli in high resolution DWI after endovascular recanalization. J Neurol 267:1401-1406. https://doi.org/10.1007/ s00415-020-09719-1

11. Liebeskind DS, Bracard S, Guillemin F et al (2018) eTICI reperfusion: defining success in endovascular stroke therapy. J Neurointerv Surg. https://doi.org/10.1136/neurintsurg-2018-014127 
12. Powers WJ, Rabinstein AA, Ackerson T et al (2018) 2018 guidelines for the early management of patients with acute ischemic stroke: a guideline for healthcare professionals from the American Heart Association/American Stroke Association. Stroke 49:e46e110

13. DGN (2015) Akuttherapie des ischämischen Schlaganfalls Rekanalisierende Therapie (Ergänzung 2015)

14. Liebeskind DS, Bracard S, Guillemin F et al (2019) eTICI reperfusion: defining success in endovascular stroke therapy. J Neurointerv Surg 11:433-438

15. Treurniet KM, LeCouffe NE, Kappelhof M et al (2021) MR CLEAN-NO IV: intravenous treatment followed by endovascular treatment versus direct endovascular treatment for acute ischemic stroke caused by a proximal intracranial occlusion-study protocol for a randomized clinical trial. Trials 22:141

16. Kaesmacher J, Boeckh-Behrens T, Simon S et al (2017) Risk of thrombus fragmentation during endovascular stroke treatment. AJNR Am J Neuroradiol 38:991-998

17. Ernst M, Boers AMM, Aigner A et al (2017) Association of computed tomography ischemic lesion location with functional outcome in acute large vessel occlusion ischemic stroke. Stroke 48: 2426-2433

18. Gratz PP, Schroth G, Gralla J et al (2015) Whole-brain susceptibility-weighted thrombus imaging in stroke: fragmented thrombi predict worse outcome. AJNR Am J Neuroradiol 36:1277-1282

19. Fischer U, Kaesmacher J, Mendes Pereira V et al (2017) Direct mechanical thrombectomy versus combined intravenous and mechanical thrombectomy in large-artery anterior circulation stroke: a topical review. Stroke 48:2912-2918
20. Broocks G, Kniep H, Kemmling A et al (2020) Effect of intravenous alteplase on ischaemic lesion water homeostasis. Eur J Neurol 27:376-383

21. Rapp JH, Pan XM, Sharp FR et al (2000) Atheroemboli to the brain: size threshold for causing acute neuronal cell death. J Vasc Surg 32: 68-76

22. Kaesmacher J, Kurmann C, Jungi $N$ et al (2020) Infarct in new territory after endovascular stroke treatment: a diffusion-weighted imaging study. Sci Rep 10:8366

23. Chueh JY, Kuhn AL, Puri AS, Wilson SD, Wakhloo AK, Gounis MJ (2013) Reduction in distal emboli with proximal flow control during mechanical thrombectomy: a quantitative in vitro study. Stroke 44:1396-1401

24. Meinel TR, Kaesmacher J, Mosimann PJ et al (2020) Association of initial imaging modality and futile recanalization after thrombectomy. Neurology 95:e2331-e2342

25. Nael K, Khan R, Choudhary G et al (2014) Six-minute magnetic resonance imaging protocol for evaluation of acute ischemic stroke: pushing the boundaries. Stroke 45:1985-1991

26. Jovin TG (2018) MRI-guided intravenous alteplase for stroke - still stuck in time. N Engl J Med 379:682-683

27. Thomalla G, Simonsen CZ, Boutitie F et al (2018) MRI-guided thrombolysis for stroke with unknown time of onset. N Engl J Med 379:611-622

28. Sheth SA, Jahan R, Gralla J et al (2015) Time to endovascular reperfusion and degree of disability in acute stroke. Ann Neurol 78:584-593

Publisher's note Springer Nature remains neutral with regard to jurisdictional claims in published maps and institutional affiliations. 